\title{
Quantum Entanglement and the Generalized Uncertainty Principle
}

\author{
Gardo Blado ${ }^{*}$, Francisco Herrera, and Joshuah Erwin \\ Department of Mathematics and Physics, College of Science and Mathematics \\ Houston Baptist University
}

7502 Fondren Rd., Houston, Texas, U.S.A

\begin{abstract}
We examine quantum gravity effects on entanglement by a straightforward application of the generalized uncertainty principle (GUP) to continuous-variable systems. In particular, we study the following cases: the modified uncertainty relation of two identical entangled particles (Rigolin, 2002), and the inseparability conditions for entangled particles in the bipartite (Duan, Giedke, Cirac and Zoller, 2000) and tripartite (van Loock and Furusawa, 2003) cases. Rigolin showed a decrease in the lower bound of the product of the uncertainties of the position and momentum for two identical entangled particles while Duan and van Loock derived inseparability conditions for EPR-like operators. In all three cases, the GUP correction resulted in a higher value of the bounds: a higher lower bound for the Rigolin's result and a higher upper bound for the inseparability condition in Duan and van Loock's relations. In Rigolin's case, the GUP correction decreased the disagreement with the Heisenberg uncertainty relation while in Duan's and Loock's case, the inseparability and entanglement conditions are enhanced. Interestingly, the GUP corrections tend to make quantum mechanical effects more pronounced.
\end{abstract}

Key words: generalized uncertainty principle, minimal length, entanglement

\footnotetext{
* Corresponding author:

Email address: gblado@hbu.edu
} 


\section{Introduction}

The formulation of a viable quantum gravity theory has long been sought by theoretical physicists as part of the effort to unify the four forces of nature: electromagnetic, weak, strong and gravitational forces. An interesting phenomenological consequence of quantum gravity theories (like string theory and loop quantum gravity) is the presence of a minimal length scale which can result from the modification of the Heisenberg uncertainty principle (HUP) to a generalized uncertainty principle (GUP) [1-11]. The HUP, $\Delta x \Delta p \geq \hbar / 2$ is changed to [9]

$\Delta x \Delta p \geq \frac{\hbar}{2}\left[1+\beta(\Delta p)^{2}+\beta\langle p\rangle^{2}\right]$

due to the modification of the commutator $[x, p]=i \hbar$ to

Equation 1: $[x, p]=i \hbar\left[1+\beta p^{2}\right]$.

It can be shown that keeping the position operator the same while changing the momentum operator as

Equation 2: $x=x_{0}, p=p_{0}\left(1+\beta p_{0}^{2}\right)$

in which $x_{0}$ and $p_{0}$ satisfy the HUP commutator, $\left[x_{0}, p_{0}\right]=i \hbar$, can give rise to the GUP. $\beta$ is called the GUP parameter which is small. Equation 2 leads to a modified Schrodinger equation $\left(p_{0}^{2} / 2 m+\right.$ $\left.\beta p_{0}^{4} / m+V\right) \psi=E \psi$ to order $\beta$ with $p_{0}=(\hbar / i) d / d x$. This has led to a number of phenomenological studies on GUP effects $[1,3,5-9,12-13]$.

In this paper we will study the GUP effects on quantum entanglement by applying the GUP commutator in Equation 1 to the entanglement-modified uncertainty relations derived by Rigolin [1415], and the inseparability conditions calculated for the bipartite system by Duan [16] and the tripartite system by Loock [17]. In section 2, we discuss the connection of entanglement and the uncertainty relations through the work of Rigolin, Duan and Loock. The essential derivations are reproduced to make the calculation of the GUP corrections in section 3 apparent. We give our conclusions in section 4.

\section{Entanglement and the Heisenberg Uncertainty Principle}

Entanglement and the uncertainty principle can be related through the HUP or variance relations [1422] and the entropic uncertainty principle [23-25]. In this paper, we will only study the HUP expressed as a variance relation.

\subsection{Modified Uncertainty Relations for Identical, Entangled Particles}

Rigolin's papers $[14,15]$ derived a new uncertainty relation involving an ensemble of $N$ identical and entangled particles. Using only physical observables and non-factorizable states for $N$ identical and entangled particles one can define the position and momentum physical observables in one dimension as, 
Equation 3: $Q=Q_{1}+Q_{2}+\cdots+Q_{N}$ and $P=P_{1}+P_{2}+\cdots+P_{N}$

where $Q_{i}$ and $P_{i}$ are the position and momentum observables of the $i$ th particle. From the uncertainty relation [27] $(\Delta A)^{2}(\Delta B)^{2} \geq\left(\frac{1}{2 i}\langle[A, B]\rangle\right)^{2}$, we get

Equation $4:(\Delta Q)^{2}(\Delta P)^{2} \geq\left(\frac{1}{2 i}\langle[Q, P]\rangle\right)^{2}$.

With the usual commutation relations,

Equation 5: $\left[Q_{i}, P_{j}\right]=i \hbar \delta_{i j},\left[Q_{i}, Q_{j}\right]=\left[P_{i}, P_{j}\right]=0$,

it follows that

Equation 6: $[Q, P]=\left[Q_{1}, P_{1}\right]+\left[Q_{2}, P_{2}\right]+\cdots+\left[Q_{N}, P_{N}\right]=N(i \hbar)$.

Equation 4 becomes

Equation 7: $(\Delta Q)^{2}(\Delta P)^{2} \geq \frac{N^{2} \hbar^{2}}{4}$

for $\mathrm{N}$ identical and entangled particles.

Let us consider two particles $(N=2)$. From

Equation 8: $(\Delta A)^{2}=\left\langle A^{2}\right\rangle-\langle A\rangle^{2}$,

we get

Equation 9: $(\Delta Q)^{2}=\left\langle Q^{2}\right\rangle-\langle Q\rangle^{2}$

where $Q$ for two particles in Equation 3 is $Q=Q_{1}+Q_{2}$. Upon expansion of the right hand side of Equation 9, and with Equation 5, we get

Equation 10: $(\Delta Q)^{2}=\left(\Delta Q_{1}\right)^{2}+\left(\Delta Q_{2}\right)^{2}+2\left\langle Q_{1} Q_{2}\right\rangle-2\left\langle Q_{1}\right\rangle\left\langle Q_{2}\right\rangle$

where we also used the definition in Equation 8. A similar calculation for the momentum operator yields

Equation 11: $(\Delta P)^{2}=\left(\Delta P_{1}\right)^{2}+\left(\Delta P_{2}\right)^{2}+2\left\langle P_{1} P_{2}\right\rangle-2\left\langle P_{1}\right\rangle\left\langle P_{2}\right\rangle$.

Using Equation 10 and Equation 11 in Equation 7, for $N=2$ particles, it follows that

Equation 12: $\left[\frac{1}{2}\left(\Delta Q_{1}\right)^{2}+\frac{1}{2}\left(\Delta Q_{2}\right)^{2}+\left\langle Q_{1} Q_{2}\right\rangle-\left\langle Q_{1}\right\rangle\left\langle Q_{2}\right\rangle\right] \times\left[\frac{1}{2}\left(\Delta P_{1}\right)^{2}+\frac{1}{2}\left(\Delta P_{2}\right)^{2}+\left\langle P_{1} P_{2}\right\rangle-\right.$ $\left.\left\langle P_{1}\right\rangle\left\langle P_{2}\right\rangle\right] \geq \frac{\hbar^{2}}{4}$.

We need to rewrite the preceding equation entirely in terms of $\Delta Q_{i}$ and $\Delta P_{i}$. Let us consider the states $\left|\varphi_{1}\right\rangle=\left(Q_{1} \pm Q_{2}\right)\left|\varphi_{2}\right\rangle$ and $\left|\varphi_{2}\right\rangle=|\psi\rangle$ in which $|\psi\rangle$ is a normalized two-particle state. We can use the Schwarz inequality in the form of $\left\langle\varphi_{1} \mid \varphi_{1}\right\rangle\left\langle\varphi_{2} \mid \varphi_{2}\right\rangle \geq\left\langle\varphi_{1} \mid \varphi_{2}\right\rangle\left\langle\varphi_{2} \mid \varphi_{1}\right\rangle$. Taking the $\left|\varphi_{1}\right\rangle=$ 
$\left(Q_{1}+Q_{2}\right)\left|\varphi_{2}\right\rangle$ case, we get $\frac{1}{2}\left(\Delta Q_{1}\right)^{2}+\frac{1}{2}\left(\Delta Q_{2}\right)^{2} \geq-\left(\left\langle Q_{1} Q_{2}\right\rangle-\left\langle Q_{1}\right\rangle\left\langle Q_{2}\right\rangle\right)$ while taking the $\left|\varphi_{1}\right\rangle=$ $\left(Q_{1}-Q_{2}\right)\left|\varphi_{2}\right\rangle$ case, we get $\frac{1}{2}\left(\Delta Q_{1}\right)^{2}+\frac{1}{2}\left(\Delta Q_{2}\right)^{2} \geq+\left(\left\langle Q_{1} Q_{2}\right\rangle-\left\langle Q_{1}\right\rangle\left\langle Q_{2}\right\rangle\right)$, hence we get $\frac{1}{2}\left(\Delta Q_{1}\right)^{2}+$ $\frac{1}{2}\left(\Delta Q_{2}\right)^{2} \geq\left|\left\langle Q_{1} Q_{2}\right\rangle-\left\langle Q_{1}\right\rangle\left\langle Q_{2}\right\rangle\right|$. Adding $\frac{1}{2}\left(\Delta Q_{1}\right)^{2}+\frac{1}{2}\left(\Delta Q_{2}\right)^{2}$ on both sides of the preceding equation, we get $\left(\Delta Q_{1}\right)^{2}+\left(\Delta Q_{2}\right)^{2} \geq \frac{1}{2}\left(\Delta Q_{1}\right)^{2}+\frac{1}{2}\left(\Delta Q_{2}\right)^{2}+\left|\left\langle Q_{1} Q_{2}\right\rangle-\left\langle Q_{1}\right\rangle\left\langle Q_{2}\right\rangle\right| \geq \frac{1}{2}\left(\Delta Q_{1}\right)^{2}+$ $\frac{1}{2}\left(\Delta Q_{2}\right)^{2}+\left\langle Q_{1} Q_{2}\right\rangle-\left\langle Q_{1}\right\rangle\left\langle Q_{2}\right\rangle$ in which the last inequality is true because of the absolute value sign. Summarizing, we have

Equation 13: $\left(\Delta Q_{1}\right)^{2}+\left(\Delta Q_{2}\right)^{2} \geq \frac{1}{2}\left(\Delta Q_{1}\right)^{2}+\frac{1}{2}\left(\Delta Q_{2}\right)^{2}+\left\langle Q_{1} Q_{2}\right\rangle-\left\langle Q_{1}\right\rangle\left\langle Q_{2}\right\rangle$.

Similarly, we get

Equation 14: $\left(\Delta P_{1}\right)^{2}+\left(\Delta P_{2}\right)^{2} \geq \frac{1}{2}\left(\Delta P_{1}\right)^{2}+\frac{1}{2}\left(\Delta P_{2}\right)^{2}+\left\langle P_{1} P_{2}\right\rangle-\left\langle P_{1}\right\rangle\left\langle P_{2}\right\rangle$.

From Equation 13 and Equation 14, we can rewrite the inequality Equation 12 entirely in terms of $\Delta Q_{i}$ and $\Delta P_{i}$ as

Equation 15: $\left[\left(\Delta Q_{1}\right)^{2}+\left(\Delta Q_{2}\right)^{2}\right] \times\left[\left(\Delta P_{1}\right)^{2}+\left(\Delta P_{2}\right)^{2}\right] \geq \frac{\hbar^{2}}{4}$.

This equation describes two identical, entangled particles. Considering the special case when $\Delta Q_{1}=$ $\Delta Q_{2}$ and $\Delta P_{1}=\Delta P_{2}$, we get $\left(\Delta Q_{i}\right)^{2}\left(\Delta P_{i}\right)^{2} \geq \frac{\hbar}{16}$ or

Equation 16: $\Delta Q_{i} \Delta P_{i} \geq \frac{\hbar}{4}, i=1,2$.

Equation 16 implies a modified uncertainty relation for entangled particles. Equation 16 shows that entangled particles decrease the uncertainty of the position and momentum. This is consistent with reference [24] which showed that increased entanglement leads to less uncertainty.

\subsection{Separability Condition for Entangled Particles, Bipartite Case}

The paper by Duan et al. [16] derived a sufficient condition for inseparability for the entanglement of bipartite systems with continuous variable states using the HUP.

Consider the density operator $\rho$ (which is a composite bipartite state with modes 1 and 2) associated with a separable quantum state. This operator can be written as

Equation 17: $\rho=\sum_{i} \eta_{i} \rho_{i 1} \otimes \rho_{i 2}, \eta_{i} \geq 0$ and $\sum_{i} \eta_{i}=1$

with $i=1,2, \ldots$ which labels the normalized states of the two modes. Duan et. al. constructed the EPRlike operators

Equation 18: $\hat{u}=|a| \hat{x}_{1}+\frac{1}{a} \hat{x}_{2}$ and $\hat{v}=|a| \hat{p}_{1}+\frac{1}{a} \hat{p}_{2}$ 
where $a$ is an arbitrary nonzero, real number and $\hat{x}_{i}$ and $\hat{p}_{j}$ obey the commutation relations in Equation $5,\left[\hat{x}_{i}, \hat{p}_{j}\right]=i \delta_{i j}, i, j=1,2$ where we set $\hbar=1$. It follows from the uncertainty relation that

Equation 19: $\left\langle\left(\Delta \hat{x}_{j}\right)^{2}\right\rangle_{i}+\left\langle\left(\Delta \hat{p}_{j}\right)^{2}\right\rangle_{i} \geq\left|\left\langle\left[\hat{x}_{j}, \hat{p}_{j}\right]\right\rangle_{i}\right|=1$ with $j=1,2$

where the symbol $\langle\cdots\rangle_{i}$ denotes averaging over the product density operator $\rho_{i 1} \otimes \rho_{i 2}$. From Equation 8 and Equation 18, we get

Equation 20: $\left\langle(\Delta \hat{u})^{2}\right\rangle_{\rho}+\left\langle(\Delta \hat{v})^{2}\right\rangle_{\rho}=\Delta^{2}+\sum_{i} \eta_{i}\langle\hat{u}\rangle_{i}^{2}-\left(\sum_{i} \eta_{i}\langle\hat{u}\rangle_{i}\right)^{2}+\sum_{i} \eta_{i}\langle\hat{v}\rangle_{i}^{2}-\left(\sum_{i} \eta_{i}\langle\hat{v}\rangle_{i}\right)^{2}$

where we define for convenience

Equation 21: $\Delta^{2} \equiv \sum_{i} \eta_{i}\left(a^{2}\left\langle\left(\Delta \hat{x}_{1}\right)^{2}\right\rangle_{i}+\frac{1}{a^{2}}\left\langle\left(\Delta \hat{x}_{2}\right)^{2}\right\rangle_{i}+a^{2}\left\langle\left(\Delta \hat{p}_{1}\right)^{2}\right\rangle_{i}+\frac{1}{a^{2}}\left\langle\left(\Delta \hat{p}_{2}\right)^{2}\right\rangle_{i}\right)$.

Rewriting Equation 21, we get

$\Delta^{2} \equiv \sum_{i} \eta_{i}\left(a^{2}\left[\left\langle\left(\Delta \hat{x}_{1}\right)^{2}\right\rangle_{i}+\left\langle\left(\Delta \hat{p}_{1}\right)^{2}\right\rangle_{i}\right]+\frac{1}{a^{2}}\left[\left\langle\left(\Delta \hat{x}_{2}\right)^{2}\right\rangle_{i}+\left\langle\left(\Delta \hat{p}_{2}\right)^{2}\right\rangle_{i}\right]\right)$. Using Equation 19, it is apparent that

Equation 22: $\Delta^{2} \equiv \sum_{i} \eta_{i}\left(a^{2}\left[\left\langle\left(\Delta \hat{x}_{1}\right)^{2}\right\rangle_{i}+\left\langle\left(\Delta \hat{p}_{1}\right)^{2}\right\rangle_{i}\right]+\frac{1}{a^{2}}\left[\left\langle\left(\Delta \hat{x}_{2}\right)^{2}\right\rangle_{i}+\left\langle\left(\Delta \hat{p}_{2}\right)^{2}\right\rangle_{i}\right]\right) \geq \sum_{i} \eta_{i}\left(a^{2}[1]+\right.$ $\left.\frac{1}{a^{2}}[1]\right)$

or $\Delta^{2} \geq\left(a^{2}+\frac{1}{a^{2}}\right) \sum_{i} \eta_{i}$ and using $\sum_{i} \eta_{i}=1$ in Equation 17

Equation 23: $\Delta^{2} \geq\left(a^{2}+\frac{1}{a^{2}}\right)$.

Summarizing so far, from Equation 20 and Equation 23, we have

Equation 24: $\left\langle(\Delta \hat{u})^{2}\right\rangle_{\rho}+\left\langle(\Delta \hat{v})^{2}\right\rangle_{\rho} \geq\left(a^{2}+\frac{1}{a^{2}}\right)+\sum_{i} \eta_{i}\langle\hat{u}\rangle_{i}^{2}-\left(\sum_{i} \eta_{i}\langle\hat{u}\rangle_{i}\right)^{2}+\sum_{i} \eta_{i}\langle\hat{v}\rangle_{i}^{2}-$ $\left(\sum_{i} \eta_{i}\langle\hat{v}\rangle_{i}\right)^{2}=\left(a^{2}+\frac{1}{a^{2}}\right)+\sum_{i} \eta_{i}\left\{\langle\hat{u}\rangle_{i}^{2}+\langle\hat{v}\rangle_{i}^{2}\right\}-\left(\sum_{i} \eta_{i}\langle\hat{u}\rangle_{i}\right)^{2}-\left(\sum_{i} \eta_{i}\langle\hat{v}\rangle_{i}\right)^{2}$.

By the Cauchy-Schwarz inequality, we can write $\left(\sum_{i} \eta_{i}\right)\left(\sum_{i} \eta_{i}\langle\hat{u}\rangle_{i}^{2}\right) \geq\left(\sum_{i} \eta_{i}\left|\langle\hat{u}\rangle_{i}\right|\right)^{2}$ or with $\sum_{i} \eta_{i}=1$ in Equation $17,\left(\sum_{i} \eta_{i}\langle\hat{u}\rangle_{i}^{2}\right) \geq\left(\sum_{i} \eta_{i}\left|\langle\hat{u}\rangle_{i}\right|\right)^{2}$ or Equation 25: $-\left(\sum_{i} \eta_{i}\left|\langle\hat{u}\rangle_{i}\right|\right)^{2} \geq-\left(\sum_{i} \eta_{i}\langle\hat{u}\rangle_{i}^{2}\right)$.

Note also that because of the absolute value sign, $\left(\sum_{i} \eta_{i}\left|\langle\hat{u}\rangle_{i}\right|\right)^{2} \geq\left(\sum_{i} \eta_{i}\langle\hat{u}\rangle_{i}\right)^{2}$ and using Equation 25 for the second inequality in the following equation,

Equation 26: $-\left(\sum_{i} \eta_{i}\langle\hat{u}\rangle_{i}\right)^{2} \geq-\left(\sum_{i} \eta_{i}\left|\langle\hat{u}\rangle_{i}\right|\right)^{2} \geq-\left(\sum_{i} \eta_{i}\langle\hat{u}\rangle_{i}^{2}\right)$.

Similarly, we can show,

Equation 27: $-\left(\sum_{i} \eta_{i}\langle\hat{v}\rangle_{i}\right)^{2} \geq-\left(\sum_{i} \eta_{i}\left|\langle\hat{v}\rangle_{i}\right|\right)^{2} \geq-\left(\sum_{i} \eta_{i}\langle\hat{v}\rangle_{i}^{2}\right)$. 
From Equation 26 and Equation 27, Equation 24 gives $\left\langle(\Delta \hat{u})^{2}\right\rangle_{\rho}+\left\langle(\Delta \hat{v})^{2}\right\rangle_{\rho} \geq\left(a^{2}+\frac{1}{a^{2}}\right)+$ $\sum_{i} \eta_{i}\left\{\langle\hat{u}\rangle_{i}^{2}+\langle\hat{v}\rangle_{i}^{2}\right\}-\left(\sum_{i} \eta_{i}\langle\hat{u}\rangle_{i}\right)^{2}-\left(\sum_{i} \eta_{i}\langle\hat{v}\rangle_{i}\right)^{2} \geq\left(a^{2}+\frac{1}{a^{2}}\right)+\sum_{i} \eta_{i}\left\{\langle\hat{u}\rangle_{i}^{2}+\langle\hat{v}\rangle_{i}^{2}\right\}-\left(\sum_{i} \eta_{i}\langle\hat{u}\rangle_{i}^{2}\right)-$ $\left(\sum_{i} \eta_{i}\langle\hat{v}\rangle_{i}^{2}\right)$ leaving only

Equation 28: $\left\langle(\Delta \hat{u})^{2}\right\rangle_{\rho}+\left\langle(\Delta \hat{v})^{2}\right\rangle_{\rho} \geq\left(a^{2}+\frac{1}{a^{2}}\right)$

for separable states involving a pair of EPR-like operators $\hat{u}$ and $\hat{v}$. From Equation 28 we can conclude that if $\left\langle(\Delta \hat{u})^{2}\right\rangle_{\rho}+\left\langle(\Delta \hat{v})^{2}\right\rangle_{\rho}<\left(a^{2}+\frac{1}{a^{2}}\right)$ then we have inseparable states. Hence the violation of Equation 28 gives us a sufficient condition for inseparability. In summary,

Equation 29: $\left\langle(\Delta \hat{u})^{2}\right\rangle_{\rho}+\left\langle(\Delta \hat{v})^{2}\right\rangle_{\rho}<\left(a^{2}+\frac{1}{a^{2}}\right)$

for inseparable, entangled states.

\subsection{Separability Condition for Entangled Particles, Tripartite Case}

In [17], Loock and Furusawa worked out a condition similar to Duan's case for the tripartite case for partially and fully separable cases. We will show the relevant parts of the derivation of the fully separable case below.

We now look at the density operator $\boldsymbol{\rho}$ of a completely separable tripartite state with modes 1, 2 and 3, namely $\rho=\sum_{i} \boldsymbol{\eta}_{\boldsymbol{i}} \boldsymbol{\rho}_{\boldsymbol{i} \mathbf{1}} \otimes \boldsymbol{\rho}_{\boldsymbol{i} \mathbf{2}} \otimes \boldsymbol{\rho}_{\boldsymbol{i} \mathbf{3}}$ with $\boldsymbol{\eta}_{\boldsymbol{i}} \geq \mathbf{0}$ and $\sum_{\boldsymbol{i}} \boldsymbol{\eta}_{\boldsymbol{i}}=\mathbf{1}$. Consider a generalization of Equation 18 for the tripartite case, $\widehat{\boldsymbol{u}}=\boldsymbol{h}_{\mathbf{1}} \widehat{\boldsymbol{x}}_{\mathbf{1}}+\boldsymbol{h}_{\mathbf{2}} \widehat{\boldsymbol{x}}_{\mathbf{2}}+\boldsymbol{h}_{\mathbf{3}} \widehat{\boldsymbol{x}}_{\mathbf{3}}$ and $\widehat{\boldsymbol{v}}=\boldsymbol{g}_{\mathbf{1}} \widehat{\boldsymbol{p}}_{\mathbf{1}}+\boldsymbol{g}_{\mathbf{2}} \widehat{\boldsymbol{p}}_{\mathbf{2}}+\boldsymbol{g}_{\mathbf{3}} \widehat{\boldsymbol{p}}_{\mathbf{3}}$ with real parameters $\boldsymbol{h}_{\boldsymbol{i}}$ and $\boldsymbol{g}_{\boldsymbol{i}}$. Using Equation 8, we expand $\left\langle(\Delta \widehat{\boldsymbol{u}})^{\mathbf{2}}\right\rangle_{\boldsymbol{\rho}}+\left\langle(\Delta \widehat{\boldsymbol{v}})^{\mathbf{2}}\right\rangle_{\boldsymbol{\rho}}$ and get [17]

Equation 30: $\left\langle(\Delta \hat{u})^{2}\right\rangle_{\rho}+\left\langle(\Delta \hat{v})^{2}\right\rangle_{\rho}=\sum_{i} \eta_{i}\left[T_{123 i}+R_{123 i}\right]+S_{i i}$

where we define

$T_{123 i} \equiv h_{1}^{2}\left\langle\left(\Delta \hat{x}_{1}\right)^{2}\right\rangle_{i}+h_{2}^{2}\left\langle\left(\Delta \hat{x}_{2}\right)^{2}\right\rangle_{i}+h_{3}^{2}\left\langle\left(\Delta \hat{x}_{3}\right)^{2}\right\rangle_{i}+g_{1}^{2}\left\langle\left(\Delta \hat{p}_{1}\right)^{2}\right\rangle_{i}+g_{2}^{2}\left\langle\left(\Delta \hat{p}_{2}\right)^{2}\right\rangle_{i}+g_{3}^{2}\left\langle\left(\Delta \hat{p}_{3}\right)^{2}\right\rangle_{i}+$ $2 h_{1} h_{2}\left(\left\langle\hat{x}_{1} \hat{x}_{2}\right\rangle_{i}-\left\langle\hat{x}_{1}\right\rangle_{i}\left\langle\hat{x}_{2}\right\rangle_{i}\right)+2 g_{1} g_{2}\left(\left\langle\hat{p}_{1} \hat{p}_{2}\right\rangle_{i}-\left\langle\hat{p}_{1}\right\rangle_{i}\left\langle\hat{p}_{2}\right\rangle_{i}\right)$,

$R_{123 i}=2 h_{1} h_{3}\left(\left\langle\hat{x}_{1} \hat{x}_{3}\right\rangle_{i}-\left\langle\hat{x}_{1}\right\rangle_{i}\left\langle\hat{x}_{3}\right\rangle_{i}\right)+2 h_{2} h_{3}\left(\left\langle\hat{x}_{2} \hat{x}_{3}\right\rangle_{i}-\left\langle\hat{x}_{2}\right\rangle_{i}\left\langle\hat{x}_{3}\right\rangle_{i}\right)+2 g_{1} g_{3}\left(\left\langle\hat{p}_{1} \hat{p}_{3}\right\rangle_{i}-\right.$ $\left.\left\langle\hat{p}_{1}\right\rangle_{i}\left\langle\hat{p}_{3}\right\rangle_{i}\right)+2 g_{2} g_{3}\left(\left\langle\hat{p}_{2} \hat{p}_{3}\right\rangle_{i}-\left\langle\hat{p}_{2}\right\rangle_{i}\left\langle\hat{p}_{3}\right\rangle_{i}\right)$ and

$S_{i i}=\sum_{i} \eta_{i}\langle\hat{u}\rangle_{i}^{2}-\left(\sum_{i} \eta_{i}\langle\hat{u}\rangle_{i}\right)^{2}+\sum_{i} \eta_{i}\langle\hat{v}\rangle_{i}^{2}-\left(\sum_{i} \eta_{i}\langle\hat{v}\rangle_{i}\right)^{2}$.

As before in section 2.2, by the Cauchy-Schwarz inequality, we can show that $S_{i i} \geq 0$. Hence, Equation 30 becomes

Equation 31: $\left\langle(\Delta \hat{u})^{2}\right\rangle_{\rho}+\left\langle(\Delta \hat{v})^{2}\right\rangle_{\rho}=\sum_{i} \eta_{i}\left[T_{123 i}+R_{123 i}\right]+S_{i i} \geq \sum_{i} \eta_{i}\left[T_{123 i}+R_{123 i}\right]$.

Since modes 1, 2 and 3 are separable, $\left\langle\hat{x}_{m} \hat{x}_{n}\right\rangle_{i}=\left\langle\hat{x}_{m}\right\rangle_{i}\left\langle\hat{x}_{n}\right\rangle_{i}$ and $\left\langle\hat{p}_{m} \hat{p}_{n}\right\rangle_{i}=\left\langle\hat{p}_{m}\right\rangle_{i}\left\langle\hat{p}_{n}\right\rangle_{i}$. Clearly $R_{123 i}=0$ and Equation 31 becomes, 
Equation 32: $\left\langle(\Delta \hat{u})^{2}\right\rangle_{\rho}+\left\langle(\Delta \hat{v})^{2}\right\rangle_{\rho} \geq \sum_{i} \eta_{i} T_{123 i}$

with

Equation 33: $T_{123 i}=h_{1}^{2}\left\langle\left(\Delta \hat{x}_{1}\right)^{2}\right\rangle_{i}+h_{2}^{2}\left\langle\left(\Delta \hat{x}_{2}\right)^{2}\right\rangle_{i}+h_{3}^{2}\left\langle\left(\Delta \hat{x}_{3}\right)^{2}\right\rangle_{i}+g_{1}^{2}\left\langle\left(\Delta \hat{p}_{1}\right)^{2}\right\rangle_{i}+g_{2}^{2}\left\langle\left(\Delta \hat{p}_{2}\right)^{2}\right\rangle_{i}+$ $g_{3}^{2}\left\langle\left(\Delta \hat{p}_{3}\right)^{2}\right\rangle_{i}$.

Note that we can write $h_{n}^{2}\left\langle\left(\Delta \hat{x}_{n}\right)^{2}\right\rangle_{i}+g_{n}^{2}\left\langle\left(\Delta \hat{p}_{n}\right)^{2}\right\rangle_{i}=\left\langle\left(\Delta\left[h_{n} \hat{x}_{n}\right]\right)^{2}\right\rangle_{i}+\left\langle\left(\Delta\left[g_{n} \hat{p}_{n}\right]\right)^{2}\right\rangle_{i}$ and similar to Equation 19, and using $\left[\hat{x}_{i}, \hat{p}_{j}\right]=i \delta_{i j}$,

Equation 34: $h_{n}^{2}\left\langle\left(\Delta \hat{x}_{n}\right)^{2}\right\rangle_{i}+g_{n}^{2}\left\langle\left(\Delta \hat{p}_{n}\right)^{2}\right\rangle_{i}=\left\langle\left(\Delta\left[h_{n} \hat{x}_{n}\right]\right)^{2}\right\rangle_{i}+\left\langle\left(\Delta\left[g_{n} \hat{p}_{n}\right]\right)^{2}\right\rangle_{i} \geq\left|\left\langle\left[h_{n} \hat{x}_{n}, g_{n} \hat{p}_{n}\right]\right\rangle_{i}\right|=$ $\left|h_{n} g_{n}\left\langle\left[\hat{x}_{n}, \hat{p}_{n}\right]\right\rangle_{i}\right|=\left|h_{n} g_{n}\right|$.

Hence from Equation 33 and Equation 34, $T_{123 i} \geq\left|h_{1} g_{1}\right|+\left|h_{2} g_{2}\right|+\left|h_{3} g_{3}\right|$. Equation 32 becomes Equation 35: $\left\langle(\Delta \hat{u})^{2}\right\rangle_{\rho}+\left\langle(\Delta \hat{v})^{2}\right\rangle_{\rho} \geq \sum_{i} \eta_{i}\left\{\left|h_{1} g_{1}\right|+\left|h_{2} g_{2}\right|+\left|h_{3} g_{3}\right|\right\}$ and with $\sum_{i} \eta_{i}=1$, $\left\langle(\Delta \hat{u})^{2}\right\rangle_{\rho}+\left\langle(\Delta \hat{v})^{2}\right\rangle_{\rho} \geq\left|h_{1} g_{1}\right|+\left|h_{2} g_{2}\right|+\left|h_{3} g_{3}\right|$.

Hence similar to the bipartite case,

Equation 36: $\left\langle(\Delta \hat{u})^{2}\right\rangle_{\rho}+\left\langle(\Delta \hat{v})^{2}\right\rangle_{\rho}<\sum_{i} \eta_{i}\left\{\left|h_{1} g_{1}\right|+\left|h_{2} g_{2}\right|+\left|h_{3} g_{3}\right|\right\}=\left|h_{1} g_{1}\right|+\left|h_{2} g_{2}\right|+\left|h_{3} g_{3}\right|$ for inseparable, entangled states.

\section{The Generalized Uncertainty Principle and Entanglement}

We now calculate the GUP corrected equations. From Equation 1, we see that the GUP-corrected commutation relation of Equation 5 are given by

Equation 37: $\left[Q_{i}, P_{j}\right]=i \hbar \delta_{i j}\left(1+\beta P_{i}^{2}\right),\left[Q_{i}, Q_{j}\right]=\left[P_{i}, P_{j}\right]=0$.

3.1. Modified Uncertainty Relations for Identical, Entangled Particles with GUP correction

It is apparent from the calculations in subsection 2.1 above that, what will change in the relations above is the right hand side of the inequality due to the extra term proportional to the $\beta$ parameter. Using Equation 37 in Equation 6 for $N=2$ particles, results in $[Q, P]=2 i \hbar+i \hbar \beta\left[P_{1}^{2}+P_{2}^{2}\right]$. Hence we get (to order $\beta$ only)

Equation 38: $(\Delta Q)^{2}(\Delta P)^{2} \geq \hbar^{2}+\hbar^{2} \beta\left[\left\langle P_{1}^{2}\right\rangle+\left\langle P_{2}^{2}\right\rangle\right]$.

The above equation replaces Equation 7 for $N=2$ particles. Let us rewrite Equation 38 in terms of $\Delta P_{i}^{2}$ with $i=1,2$. From Equation 8 , we can write $\left\langle P_{i}^{2}\right\rangle=\left(\Delta P_{i}\right)^{2}+\left\langle P_{i}\right\rangle^{2}$. Hence Equation 38 becomes $(\Delta Q)^{2}(\Delta P)^{2} \geq \hbar^{2}+\hbar^{2} \beta\left[\left(\Delta P_{1}\right)^{2}+\left(\Delta P_{2}\right)^{2}+\left\langle P_{1}\right\rangle^{2}+\left\langle P_{2}\right\rangle^{2}\right] \geq \hbar^{2}+\hbar^{2} \beta\left[\left(\Delta P_{1}\right)^{2}+\left(\Delta P_{2}\right)^{2}\right]$. It follows that

Equation 39: $(\Delta Q)^{2}(\Delta P)^{2} \geq \hbar^{2}+\hbar^{2} \beta\left[\left(\Delta P_{1}\right)^{2}+\left(\Delta P_{2}\right)^{2}\right]$.

One can retrace the steps above using Equation 39 to get the GUP-corrected Equation 15. 
Equation 40: $\left[\left(\Delta Q_{1}\right)^{2}+\left(\Delta Q_{2}\right)^{2}\right] \times\left[\left(\Delta P_{1}\right)^{2}+\left(\Delta P_{2}\right)^{2}\right] \geq \frac{\hbar^{2}}{4}+\frac{\hbar^{2}}{4} \beta\left(\left(\Delta P_{1}\right)^{2}+\left(\Delta P_{2}\right)^{2}\right)$

which apparently reduces to Equation 15 when $\beta=0$. With the special case when $\Delta Q_{1}=\Delta Q_{2}$ and $\Delta P_{1}=\Delta P_{2}$, we get $\left(\Delta Q_{i}\right)^{2}\left(\Delta P_{i}\right)^{2} \geq \frac{\hbar}{16}\left(1+2 \beta\left(\Delta P_{i}\right)^{2}\right)$ which can be rewritten as (to the order $\beta$ )

Equation 41: $\Delta Q_{i} \Delta P_{i} \geq \frac{\hbar}{4}+\Delta_{G U P}$, with $\Delta_{G U P} \equiv \frac{\hbar}{4} \beta\left(\Delta P_{i}\right)^{2} \geq 0, i=1,2$.

Rigolin's motivation $[14,15]$ in deriving Equation 16 is to give a possible alternative explanation for the experimental results of Kim and Shih [28] which seem to imply a violation of the uncertainty principle in that $\Delta y \Delta p_{y}<\hbar$. The presence of the GUP correction puts a constraint upon which certain states of two identical and entangled particles may or may not violate the uncertainty principle. Using the form of the HUP in section $1, \Delta x \Delta p \geq \hbar / 2$, if $\Delta_{G U P} \geq \frac{\hbar}{4}$ or $\beta\left(\Delta P_{i}\right)^{2} \geq 1$ then there is no disagreement with the uncertainty principle while $\Delta_{G U P} \leq \frac{\hbar}{4}$ or $\beta\left(\Delta P_{i}\right)^{2} \leq 1$ will cause a disagreement from the uncertainty principle.

From [28] and [29], one can estimate the $\Delta P_{i}$ of the entangled photon along the $y$ axis to be $\Delta P_{i} \sim \frac{h}{2 \Delta y}$ where $\Delta y=0.16 \mathrm{~mm}$. Apparently, with a small $\beta, \beta\left(\Delta P_{i}\right)^{2} \leq 1$ and hence this causes a disagreement with the uncertainty principle as similar to Rigolin's result. However, since the $\Delta_{G U P}$ is positive in Equation 41, GUP effects increase the lower bound of the product of the variances and lessens the disagreement with the HUP for identical entangled particles. In [15] it was shown that as the number $N$ of identical entangled particles increase, the lower bound of the product of the position and momentum uncertainties decreases approaching zero as $N$ becomes very large, moving towards classicality. However, the presence of the GUP correction reduces the tendency towards classicality (hence making it more quantum mechanical) as compared to the non-GUP case for a large number of entangled identical particles.

\subsection{Separability Condition for Entangled Particles, Bipartite Case with GUP Correction}

From Equation 1, we have $\left[x_{i}, p_{i}\right]=i \hbar\left(1+\beta p_{i}^{2}\right)$. Using this equation, the GUP-corrected Equation 19 is (with $\hbar=1),\left\langle\left(\Delta \hat{x}_{j}\right)^{2}\right\rangle_{i}+\left\langle\left(\Delta \hat{p}_{j}\right)^{2}\right\rangle_{i} \geq\left|\left\langle\left[\hat{x}_{j}, \hat{p}_{j}\right]\right\rangle_{i}\right|=\left|1+\beta\left\langle\hat{p}_{j}^{2}\right\rangle_{i}\right|$. Since the quantity inside the absolute value is positive, we have,

Equation $42:\left\langle\left(\Delta \hat{x}_{j}\right)^{2}\right\rangle_{i}+\left\langle\left(\Delta \hat{p}_{j}\right)^{2}\right\rangle_{i} \geq 1+\beta\left\langle\hat{p}_{j}^{2}\right\rangle_{i}$.

From Equation 42, we get the GUP-corrected Equation 22 as $\Delta^{2} \equiv \sum_{i} \eta_{i}\left(a^{2}\left[\left\langle\left(\Delta \hat{x}_{1}\right)^{2}\right\rangle_{i}+\left\langle\left(\Delta \hat{p}_{1}\right)^{2}\right\rangle_{i}\right]+\right.$ $\left.\frac{1}{a^{2}}\left[\left\langle\left(\Delta \hat{x}_{2}\right)^{2}\right\rangle_{i}+\left\langle\left(\Delta \hat{p}_{2}\right)^{2}\right\rangle_{i}\right]\right) \geq \sum_{i} \eta_{i}\left(a^{2}\left[1+\beta\left\langle\hat{p}_{1}^{2}\right\rangle_{i}\right]+\frac{1}{a^{2}}\left[1+\beta\left\langle\hat{p}_{2}^{2}\right\rangle_{i}\right]\right)$ or

Equation 43: $\Delta^{2} \geq\left(a^{2}+\frac{1}{a^{2}}\right)+\beta f\left(\hat{p}_{1}, \hat{p}_{2}\right)$ where $f\left(\hat{p}_{1}, \hat{p}_{2}\right) \equiv \sum_{i} \eta_{i}\left[a^{2}\left\langle\hat{p}_{1}^{2}\right\rangle_{i}+\frac{1}{a^{2}}\left\langle\hat{p}_{2}^{2}\right\rangle_{i}\right]$ 
Hence the GUP-corrected Equation 28 is

Equation 44: $\left\langle(\Delta \hat{u})^{2}\right\rangle_{\rho}+\left\langle(\Delta \hat{v})^{2}\right\rangle_{\rho} \geq\left(a^{2}+\frac{1}{a^{2}}\right)+\Delta_{G U P}$ where $\Delta_{G U P} \equiv \sum_{i} \eta_{i}\left[a^{2}\left\langle\hat{p}_{1}^{2}\right\rangle_{i}+\frac{1}{a^{2}}\left\langle\hat{p}_{2}^{2}\right\rangle_{i}\right] \geq$ 0 .

The GUP-corrected Equation 29 is

Equation 45: $\left\langle(\Delta \hat{u})^{2}\right\rangle_{\rho}+\left\langle(\Delta \hat{v})^{2}\right\rangle_{\rho}<\left(a^{2}+\frac{1}{a^{2}}\right)+\Delta_{G U P}$

for inseparable states where $\Delta_{G U P}$ is given in Equation 44. Comparing Equation 29 and Equation 45, with $\Delta_{G U P} \geq 0$, the upper bound for inseparability is increased, increasing the range for inseparability and hence enhancing the entanglement of two-party continuous variable states.

\subsection{Separability Condition for Entangled Particles, Tripartite Case with GUP Correction}

Turning now to the tripartite case of section 2.3, from Equation 34,

Equation 46: $h_{n}^{2}\left\langle\left(\Delta \hat{x}_{n}\right)^{2}\right\rangle_{i}+g_{n}^{2}\left\langle\left(\Delta \hat{p}_{n}\right)^{2}\right\rangle_{i}=\left\langle\left(\Delta\left[h_{n} \hat{x}_{n}\right]\right)^{2}\right\rangle_{i}+\left\langle\left(\Delta\left[g_{n} \hat{p}_{n}\right]\right)^{2}\right\rangle_{i} \geq\left|\left\langle\left[h_{n} \hat{x}_{n}, g_{n} \hat{p}_{n}\right]\right\rangle_{i}\right|=$ $\left|h_{n} g_{n}\left\langle\left[\hat{x}_{n}, \hat{p}_{n}\right]\right\rangle_{i}\right|=\left|h_{n} g_{n}\left\langle 1+\beta \hat{p}_{n}^{2}\right\rangle_{i}\right|=\left|h_{n} g_{n}\left(1+\beta\left\langle\hat{p}_{n}^{2}\right\rangle_{i}\right)\right|$.

Hence from Equation 33 and Equation 46, we have $T_{123 i} \geq\left|h_{1} g_{1}\left(1+\beta\left\langle p_{1}^{2}\right\rangle_{i}\right)\right|+\mid h_{2} g_{2}(1+$ $\left.\beta\left\langle p_{2}^{2}\right\rangle_{i}\right)|+| h_{3} g_{3}\left(1+\beta\left\langle p_{3}^{2}\right\rangle_{i}\right) \mid$. From Equation 32, we have

Equation 47: $\left\langle(\Delta \hat{u})^{2}\right\rangle_{\rho}+\left\langle(\Delta \hat{v})^{2}\right\rangle_{\rho} \geq \sum_{i} \eta_{i}\left\{\left|h_{1} g_{1}\left(1+\beta\left\langle p_{1}^{2}\right\rangle_{i}\right)\right|+\left|h_{2} g_{2}\left(1+\beta\left\langle p_{2}^{2}\right\rangle_{i}\right)\right|+\mid h_{3} g_{3}(1+\right.$ $\left.\left.\beta\left\langle p_{3}^{2}\right\rangle_{i}\right) \mid\right\}$

for completely separable states. Therefore, for inseparable states,

Equation 48: $\left\langle(\Delta \hat{u})^{2}\right\rangle_{\rho}+\left\langle(\Delta \hat{v})^{2}\right\rangle_{\rho}<\sum_{i} \eta_{i}\left\{\left|h_{1} g_{1}\left(1+\beta\left\langle p_{1}^{2}\right\rangle_{i}\right)\right|+\left|h_{2} g_{2}\left(1+\beta\left\langle p_{2}^{2}\right\rangle_{i}\right)\right|+\mid h_{3} g_{3}(1+\right.$ $\left.\left.\beta\left\langle p_{3}^{2}\right\rangle_{i}\right) \mid\right\}$

Comparing Equation 48 and Equation 36, the upper bound for inseparability is increased, increasing the range for inseparability and hence enhancing the entanglement of three-party continuous variable states.

\section{Conclusions}

By a straightforward application of the modified commutation relation (Equation 1), we have shown how the generalized uncertainty principle or the presence of minimal length affect entanglement. In all three cases, the bounds were increased by GUP effects in such a way as to make the quantum effects more pronounced. In the first case an increase in the lower bound makes the disagreement with the uncertainty principle less such that there is less classicality when the number of identical entangled particles becomes large. In the second and third cases, an increase in the upper bound of the inseparability condition increases the range of inseparability and hence enhances entanglement. These results are in agreement with reference [30] in which it was shown that nonclassicality and entanglement are enhanced by increasing the noncommutativity of the underlying space (an alternate 
way of introducing minimal length similar to the GUP). Similarly, in ref [31], it is shown that the entanglement of Gaussian states can be induced by a noncommutative phase space scenario.

In this paper, we confined ourselves in the study of GUP effects on continuous variable systems with the variance form of the uncertainty principle $(\Delta x \Delta p \geq \hbar / 2)$. Further studies on the effect of minimal length and GUP on continuous variable systems can be done for multipartite systems [17, 20, 22].

An interesting study of the effects of minimal length and GUP on entanglement can be made via the entropic uncertainty principle [23-25, 33-35]. This will be the topic of a future publication.

\section{Acknowledgements}

The authors would like to thank Rafael Sepulveda of Houston Baptist University for his work on the initial stages of this paper.

\section{References:}

[1] G. Blado, T. Prescott, J. Jennings, J. Ceyanes, R. Sepulveda, Eur. J. Phys. 37 (2016) 025401

[2] A. A. Ali, E.C. Vagenas, Europhys. Lett. 112 (2015) 20005. arXiv: 1510.06365[gr-qc]

[3] G. Blado, C. Owens, V. Meyers, Eur. J. Phys. 35 (2014) 065011

[4] S. Hossenfelder, Living Rev. Relativ. 16 (2013) 2-90

[5] M. Sprenger, P. Nicolini, M. Bleicher, Eur. J. Phys. 33 (2012) 853-862

[6] A. F. Ali, S. Das, E. C. Vagenas, Phys. Rev. D 84 (2011) 044013-044022

[7] S. Das, E. C. Vagenas, Can. J. Phys. 87 (2009) 233-240

[8] A. F. Ali, S. Das, E. C. Vagenas, Phys. Lett. B 678 (2009) 497-499

[9] A. Kempf, G. Mangano, R. B. Mann, Phys. Rev. D 52 (1995) 1108-1118

[10] L. J. Garay, Int. J. Mod. Phys. A 10 (1995) 145-166

[11] M. Maggiore, Phys. Lett B 304 (1993) 65-69. arxiv:hep-th/9301067

[12] K. Nozari, T. Azizi, Gen. Relativ. Gravit. 38 (2006) 735-742

[13] J. Vahedi, K. Nozari, P. Pedram, Gravit. Cosmol. 18 (2012) 211-215

[14] G. Rigolin, Found. Phys. Lett. 15 (2002) 293-298. arXiv: quant-ph/0008100 [quant-ph]

[15] G. Rigolin G (2001). arXiv: quant-ph/0105057 [quant-ph]

[16] L. Duan, G. Giedke, J. I. Cirac, P. Zoller, Phys. Rev. Lett. 84 (2000) 2722-2725

[17] P. van Loock, A. Furusawa, Phys Rev A 67 (2003) 052315

[18] A.C. de la Torre, P. Catuogno, S. Ferrando, Foundations of Physics Letters 2 (1989) 235-244

[19] R. Simon, Phys. Rev. Lett. 84 (2000) 2726-2729

[20] S.Mancini, V. Giovannetti, D. Vitali, P. Tombesi, Phys. Rev. Lett. 88 (2002) 120401

[21] V. Giovannetti, S. Mancini, D. Vitali, P. Tombesi, Phys. Rev. A 67 (2003) 022320

[22] J. Y. Zeng, Y. A. Lei , S. Y. Pei, X. C. Zeng. arXiv: 1306.3325 [quant-ph]

[23] J. Oppenheim, S. Wehner, Science 330 (2010) 1072-1074

[24] M. Berta, P. J. Coles, S. Wehner, Phys. Rev. A 90 (2014) 062127. arXiv: 1302.5902 [quant-ph]

[25] P.J. Coles, M. Berta, M. Tomamichel, S. Wehner, Rev. Mod. Phys. 89 (2017) 015002. arXiv: 1511.04857 [quant-ph] 
[26] R. Horodecki, P. Horodecki, M. Horodecki, K. Horodecki, Rev. Mod. Phys. 81 (2009) 865942. arXiv:quant-ph/0702225

[27] D. J. Griffiths, Introduction to Quantum Mechanics, Prentice-Hall, (2005)

[28] Y. H. Kim, Y. Shih, Found. Phys. 29 (1999) 1849

[29] R. P. Feynman, R. B. Leighton, M. L. Sands, The Feynman Lectures on Physics, volume III, Addison Wesley, (1963)

[30] S. Dey, A. Fring, V. Hussin, Int. J. Mod. Phys. B 31 (2016) 1650248. arXiv: 1506.08901 [quant-ph]

[31] A. E. Bernardini, C. Bastos, O. Bertolami, N. C. Dias, J. N. Prata, J. Phys. Conf. Ser. 626 (2015) 012046. arXiv: 1410.8752 [quant-ph]

[32] M. Gessner, L. Pezze, A. Smerzi. arXiv: 1702.08413 [quant-ph]

[33] P. Pedram, Adv. High Energy Phys. 2016 (2016) 5101389. arXiv: 1603.06869 [quant-ph]

[34] A. E. Rastegin, Annals Phys. 382 (2017) 170-180. arXiv: 1607.08512 [quant-ph]

[35] L.Y. Hsu, S. Kawamoto, W.Y. Wen. arXiv: 1605.04783 [hep-th] 